\title{
Size distribution of raindrops
}

To the Editor: Villermaux and Bossa ${ }^{1}$ (hereafter referred to as VB) presented a model to describe bag break-up of large drops (diameter $d \geq 6 \mathrm{~mm}$ ), which yields a drop size distribution consistent with Marshall-Palmer's distribution for rainfall ${ }^{2}$. Based on this result, and a laboratory demonstration of bag break-up, VB propose that the equilibrium drop size distribution of natural rainfall is the result of the spontaneous fragmentation of single large drops ( $d \geq 6 \mathrm{~mm})$, and that the contribution of drop-drop interactions such as coalescence and collisional break-up is negligible. This proposition is ill-founded. First, the equilibrium Marshall-Palmer distribution is not representative of the variability and transient nature of natural rainfall ${ }^{3}$. That the VB break-up model yields the Marshall-Palmer distribution is therefore not sufficient to establish that it is physically relevant. Second, field observations show that the number concentration of large drops $(d \geq 6 \mathrm{~mm})$ if observed is extremely small ${ }^{4,5}$. VB do not address the physics required to explain the population dynamics of such large drops consistent with the duration, intensity and microstructure of natural rainfall ${ }^{6}$. Third, the laboratory experiments reported by VB do not replicate the free-fall conditions of natural rainfall ${ }^{7,8}$.

Whereas evidence of bag break-up is limited ${ }^{9}$, collisional disk break-up that results from the fragmentation of a large drop originated by the near head-on collision of two drops is well documented ${ }^{10}$. The transient shape of the large drop evolves to form a 'bag' after collision, and then a 'disk' before breaking to yield a large number of small drops. VB speculate that collisional break-up is unlikely. However, the mean free path for drop-drop collision is of the same order of magnitude as the average distance a drop has to fall before experiencing spontaneous break-up ${ }^{3}$. Furthermore, spontaneous break-up occurs only once for each (rare) large drop, whereas many interactions can occur among a wide size range of small drops in their downward trajectory. Indeed, surveys of laboratory experiments under free-fall conditions ${ }^{7-9}$ indicate that collisional disk break-up is responsible for up to $20 \%$ of all break-up events ${ }^{7,8}$ (other types of break-up being filament and sheet break-up ${ }^{7,8,10}$ ), whereas none or singular observations of spontaneous break-up are reported. VB's thesis that single-drop fragmentation determines the size distribution of raindrops is in conflict with field and laboratory evidence reported so far.

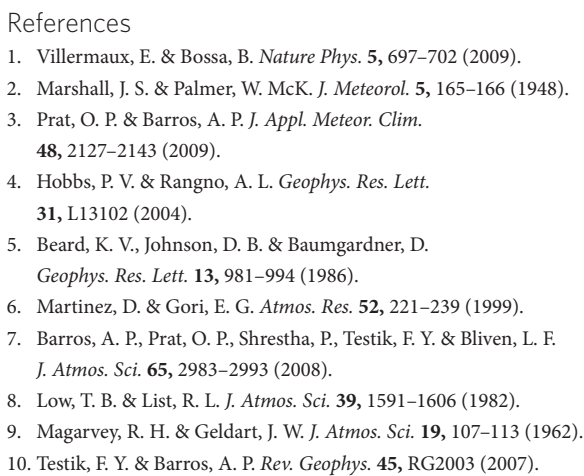

Ana P. Barros ${ }^{1 \star}$, Olivier P. Prat ${ }^{1}$ and Firat Y. Testik ${ }^{2}$

${ }^{1}$ Civil and Environmental Engineering Department, Pratt School of Engineering, Box 90287, 2457 CIEMAS Fitzpatrick Building, Duke University, Durham, North Carolina 27708, USA, ${ }^{2}$ Civil Engineering Department, College of Engineering and Science, Clemson University, Clemson, South Carolina 29634, USA.

*e-mail: barros@duke.edu
Villermaux and Bossa reply: Collisions between drops, when enforced by an artificially confined channel (as opposed to a free turbulent air jet with turbulence levels much more representative of the medium at a cumulus base than those achieved within a potential steam, carefully prepared in a laboratory wind tunnel) may influence their size distribution. It is also true that collisions do occur in real precipitations, and can mediate a splash-like interaction. When it actually occurs, the phenomenon is very similar to bag break-up ${ }^{1}$ : the formation of a corrugated toroidal rim (disk), suffering a capillary instability producing thinner drops; their size distribution is likely to be similar. That scenario would also be equally consistent, as ours is, with the observation that apparent fall velocities can be larger than the terminal velocity for a given size ${ }^{2}$. Fragments have, transitorily, the same velocity as the mother drop(s) before relaxing towards their equilibrium fall velocity.

But random collisions are, at best, a twoparticle process, necessarily rare compared with the deterministic (and fast ${ }^{3}$ ) break-up of big liquid globules found at the clouds' base $^{4}$ (hence their absence in the falling rain), as well as not always being efficient for break-up ${ }^{5}$ : a collision may result in aggregation (forming a bigger drop), in nothing (drops just bouncing off each other), or in splash (forming fragments). Splash occurs under even rarer, very special conditions of trajectory alignment and relative velocities. Even at the scale of a burst drop, fragments are so dilute that they never interact. In any case, the effect of these decorative events is completely screened by spontaneous break-up, which in itself contains the whole spectrum of drop sizes, and coincides quantitatively with the Marshall-Palmer distribution, a widely accepted fit for the drop size distribution in rain ${ }^{6}$.

One swallow does not a summer make: collisions are one among the many phenomena in the rich zoology that accompanies rainfall ${ }^{7}$. Historically, collisions have been invoked for mediating aggregation, or break-up, or both ${ }^{8}$, but in spite of their fortuitous occurrence in ad hoc laboratory settings ${ }^{9}$, their rarity in nature disqualifies them as principal actors, whereas the spontaneous break-up model offers a quantitative understanding for the structure of rainfall ${ }^{3}$.

\footnotetext{
References

1. Villermaux, E. Annu. Rev. Fluid Mech. 39, 419-446 (2007).

2. Montero-Martinez, G., Kostinski, A. B., Shaw, R. A. \& Garcia-Garcia, F. Geophys. Res. Lett. 36, L11818 (2009)

3. Villermaux, E. \& Bossa, B. Nature Phys. 5, 697-702 (2009).

4. Hobbs, P. V. \& Rangno, A. L. Geophys. Res. Lett. 31, L13102 (2004).

5. Qian, J. \& Law, C. J. Fluid Mech. 331, 59-80 (1997).

6. Pruppacher, H. R. \& Klett, J. D. Microphysics of Clouds and Precipitation (Kluwer Academic Publishers, 1997). 7. Mason, B. J. The Physics of Clouds (Clarendon Press, 1971).

8. Srivastava, R. J. Atmos. Sci. 28, 410-415 (1971).

9. Testik, F. Y. \& Barros, A. P. Rev. Geophys. 45, 1-21 (2007).
}

E. Villermaux ${ }^{1,2 \star}$ and B. Bossa'

${ }^{1}$ Aix-Marseille Université, IRPHE, 13384

Marseille Cedex 13, France, 2 Institut

Universitaire de France, 103 bd Saint Michel, 75005 Paris, France.

*e-mail: villermaux@irphe.univ-mrs.fr 